\title{
TACKLING THE LEARNING OF MATHEMATICS
}

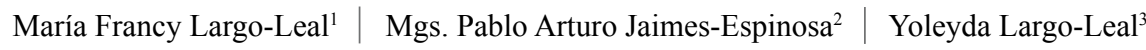

Forma de citar: LARGO-LEAL María, JAIMES ESPINOSA Pablo, LARGO-LEAL Yoleyda. Abordando el aprendizaje de las matemáticas. Eco.Mat. 2014; 5(1): 60-65.

Recibido:

Abril 22 de 2014

Aceptado:

Julio 10 de 2014

\section{RESUMEN}

Los estudiantes en su proceso de formación dentro de la integralidad que ofrece la Institución Educativa la Frontera, de acuerdo con su Proyecto Educativo Institucional PEI, deben cumplir con los estándares y competencias según los lineamientos del Ministerio de Educación Nacional MEN. Tomando en particular el área de matemáticas, genera en el estudiante expectativas en la aprehensión del conocimiento el cual evidencia algunas dificultades que merecen ser analizadas para promover orientaciones que direccionen la superación y el gusto por las mismas.

El cambio de nivel de básica primaria a secundaria concretamente, de quinto a sexto grado ha generado en el estudiante inseguridad en el desarrollo de procesos de la estructura curricular de la matemática; ubicar el referente metodológico del pensamiento matemático vs. el rendimiento académico sin señalamiento de implicados en el fracaso escolar: (docentes, estudiantes, padres de familia, entre otros).

El contexto sociopolítico particular del sector de la Parada; frontera activa con la República Bolivariana de Venezuela, considerada la frontera más dinámica de Latinoamérica por el comercio bilateral, lo que hace imprescindible el uso de las competencias matemáticas, como un aprendizaje inherente a la cotidianidad.

${ }^{1}$ Doctorando en educación. Universidad Pedagógica Experimental Libertador. Institución Educativa General Santander - Villa del Rosario. E mail: francy_286@hotmail. \section{com}

${ }^{2}$ Doctorando en educación. Universidad Pedagógica Experimental Libertador. Institución Educativa General Santander - Villa del Rosario. E mail: arturo.jaimes@hotmail.com

${ }^{3}$ Doctorando en educación. Universidad Pedagógica Experimental Libertador. Institución Educativa La Frontera - Villa del Rosario.

Email: yoleida1@hotmail.com
Palabras clave: Transposición, enseñanza, aprendizaje, rendimiento escolar.

\section{SUMMARY}

Students in the process of formation within the comprehensive educational institution that offers the Frontier, according to the Institutional Educational Project PEI, must meet the standards and competencies as outlined by the Ministry of National Education MEN. Taking in particular the area knowledge in which evidence some problems that deserve analyzed routed guidelines to promote improvement and a taste for them. of mathematics, student expectations generated in the apprehension of 
The change in level of elementary school to secondary specifically, from fifth to sixth grade student has created uncertainty in the development process of the curricular structure of mathematics; locate the methodological reference of mathematical thinking vs. academic performance without marking of school failure involved ( teachers, students, parents, etc.).

The particular sociopolitical context save sector, active border with the Bolivarian Republic of Venezuela, considered the most dynamic in Latin America for the bilateral border trade, which makes essential use of mathematics skills, as an inherent learning every day.

Keywords: Transposition, teaching, learning, school performance

\section{INTRODUCCIÓN}

$\mathrm{E}$ 1 ser humano en su formación está predispuesto a tomar las vivencias positivas de aquellas actividades y quehacer de su contexto sociocultural y del ambiente del entorno educativo que orientan los diversos lineamientos de las programaciones curriculares emanadas por el Ministerio de Educación Nacional MEN. En una localidad se retoman aquellas actividades que son inherentes al rol social con énfasis en lo socioeconómico. Villa del Rosario por ser un municipio estratégico de frontera; el comercio organizado, el informal y aquellas actividades relativas a las anteriores que generan "trabajo, empleo", como un medio de subsistencia de la población focalizada; en estas situaciones no hay distinción de clase social alta, hasta llegar a la marginalidad. "Todos necesitan de todos"; cuando el valor cambiario en países vecinos, la divisa tiene una diferencia en poder adquisitivo de la moneda; toda la población necesita el uso de las "matemáticas". Es aquí donde la operacionalización y práctica toma delantera con relación a otras áreas fundamentales del conocimiento.

La escuela es quién orienta e induce el aprendizaje de la matemática con todos los lineamientos propuestos por el M.E.N. Algunas dificultades sean de actitud por parte de los estudiantes frente a las metodologías y conceptos relacionados con los procesos que en la cotidianidad traen algunas reflexiones concluyentes como facilidad o dificultad para aprehensión del conocimiento y practica en un ambiente fronterizo. Tomando como punto de referencia la Institución Educativa La Frontera, ubicada en el municipio de Villa del Rosario a escasos metros de la República Bolivariana de Venezuela; con característica de atención para una población estudiantil flotante, por las expectativas del trabajo generadas a padres de familia que muchas veces son positivas o dejan algunos $\sin$ sabores en la comunidad. Un reflejo latente en el estudiantado con relación explicita de las matemáticas, se encuentra en el paso de educación básica primaria a secundaria; por consiguiente es pertinente dar apertura a una investigación en la línea lógico-matemática, partiendo de un diagnóstico para encontrar la relación entre el discurso matemático, la transposición y el proceso de enseñanzaaprendizaje de las matemáticas.

Según Chevallard la transposición, es un proceso que se realiza en las prácticas de enseñanza de los docentes, pero esto no la agota. Para describir este proceso es necesario distinguir el movimiento que lleva de un saber -en tanto objeto producido por la cultura- aun saber a enseñar, del que transforma este saber a enseñar en un saber 
No. 1

Enero-Diciembre 2014 ISSN 1794-8231

PP: 60-65 enseñado en un nivel de diseño, por un lado y en el de ejecución por otro. Es decir que es parte del curriculum con respecto al último movimiento. En esto es clave el análisis social, cultural e histórico y político concreto de la coyuntura correspondiente donde se produce la transformación de los saberes a enseñar. (Darcy Colmenares, 2004). Saber disciplinario (lo enciclopédico) - Docente Enseñanza (aprendizaje).

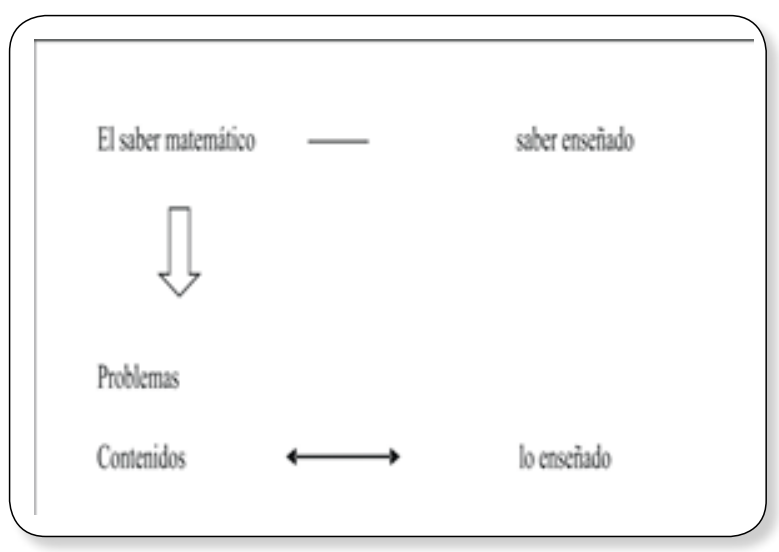

Esquema 1 (tomado de Chevallard, 1985, pág. 39)

(Chevallard, 1991: 23) Para el autor el "saber enseñado" es reproducción de saber y el "saber sabio" es producción del saber, de allí se desprenden objetos de saber de lo didáctico y de la Didáctica. En el "sistema didáctico" hay tres lugares: docente, alumno y saber.

Para Miguel de Guzmán (2007), uno de los grandes matemáticos del siglo $\mathrm{XX}$, en su interés por mejorar la Educación Matemática, señalaba que "es necesario romper, con todos los medios, la idea preconcebida, y fuertemente arraigada en nuestra sociedad, proveniente con probabilidad de bloqueos iniciales en la niñez de muchos, de que la matemática es necesariamente aburrida, abstrusa, inútil, inhumana y muy difícil". Es evidente que el rendimiento académico está relacionado con los procesos de aprendizaje. Además, Alonso et al. (1999: 61) Señalan que el panorama de trabajos sobre rendimiento académico y Estilos de Aprendizaje es muy amplio y después de analizar las distintas investigaciones se llega a la conclusión de que parece suficientemente probado que los estudiantes aprenden con más efectividad cuando se les enseña con sus Estilos de Aprendizaje predominantes.

Es de vital importancia indagar el estado emocional de los estudiantes de sexto grado con relación al área. Aprovechando que la Institución La Frontera presta el servicio educativo oficial a 130 estudiantes de sexto grado. En cierto modo era de esperar ya que dentro del terreno educativo, encontramos argumentos (Goleman 1996: 301) que sostienen que el éxito escolar del niño tiene mucho que ver con factores emocionales o sociales, en ocasiones incluso más que con sus acciones o sus capacidades intelectuales. Prueba de ello es que los ingredientes de los que depende el rendimiento escolar están íntimamente vinculados con la inteligencia emocional: confianza, curiosidad, intencionalidad, autocontrol, relación, capacidad de comunicación y cooperación.

Inteligencia social e interacción. La Inteligencia social es la capacidad que tiene todo ser humano en cada acción que realiza para relacionarse con otras personas, en beneficio de su supervivencia.

Daniel Goleman, dice que: "El enfoque sobre el conocimiento de las relaciones deja de lado las habilidades no cognitivas esenciales tales como la empatía primaria y la sincronía, e ignora capacidades como la preocupación. Una perspectiva exclusivamente cognitiva hace a un lado el esencial pegamento social cerebro a cerebro que constituye la base para cualquier interacción. Todo el espectro de habilidades de la inteligencia social abraza las aptitudes tanto del camino alto como del bajo. Tanto el concepto como sus mediciones omiten demasiadas sendas del camino bajo $y$, así, excluyen los talentos sociales que han sido claves para la supervivencia humana (Inteligencia social, 2006, pág. 151).

En correspondencia con la investigación, en el 2005 aparece publicado un artículo titulado tendencias y propuestas para la enseñanza de la matemática en sexto grado, en la revista Educere v.9 n.29; escrito por Miriam Terán 
de Serrentino y Lizabeth Pachano Rivera. Está investigación tuvo como propósito determinar la aplicabilidad de un conjunto de estrategias constructivistas para la enseñanza y el aprendizaje de la matemática en sexto grado de educación básica.

\section{METODOLOGÍA}

Estudio realizado en la Institución Educativa la Frontera, sector La Parada, municipio de Villa del Rosario; de carácter oficial, presta el servicio educativo en los niveles de educación preescolar, básica y media; con una cobertura de 1252 estudiantes atendidos por 42 docentes. Se apoya en la metodología tipo cuantitativo, con enfoque descriptivo y de carácter exploratorio. Población de tendencia flotante, con estratificación socioeconómica en los niveles 1 y 2 del SISBEN; presentándose gran porcentaje de vulnerabilidad por desplazamiento, pobreza, damnificados, contrabando, descomposición familiar y violencia entre otros. La población estuvo integrada por 130 jóvenes de Sexto Grado, distribuidos en cuatro grupos; muestra representativa de 30 estudiantes con rendimiento superior, alto, básico y bajo en el área de matemáticas; técnica muestreo dirigido.

La técnica utilizada para recolectar la información; encuesta de pregunta cerrada con opción de múltiple respuesta; en el procesamiento y análisis estadístico se utilizó el programa SPSS, las diferentes variables analizadas en el estudio fueron: género, gusto, temario, asesoría, práctica y aprendizaje de las matemáticas. El estudio enfatiza la apreciación de los estudiantes respecto del ambiente escolar e interacción pedagógica para el aprendizaje de las matemáticas.

\section{RESULTADOS Y DISCUSIÓN}

En el mes de Septiembre del 2012; se presenta la información con las diferentes variables analizadas en el estudio, el cual busca conocer la apreciación de los estudiantes al respecto del aprendizaje de las matemáticas. Hay que hacer notar, que el $63,3 \%$ de los encuestados es de género masculino y el $36.7 \%$ corresponde al femenino. Al indagar por el gusto de las matemáticas, se evidencia según la gráfica 1 , que al $50 \%$ le gusta mucho, al $43,3 \%$ regular, al 3,3\% casi nada, y el restante 3,3\% Ns/Nr (No sabe, no responde).

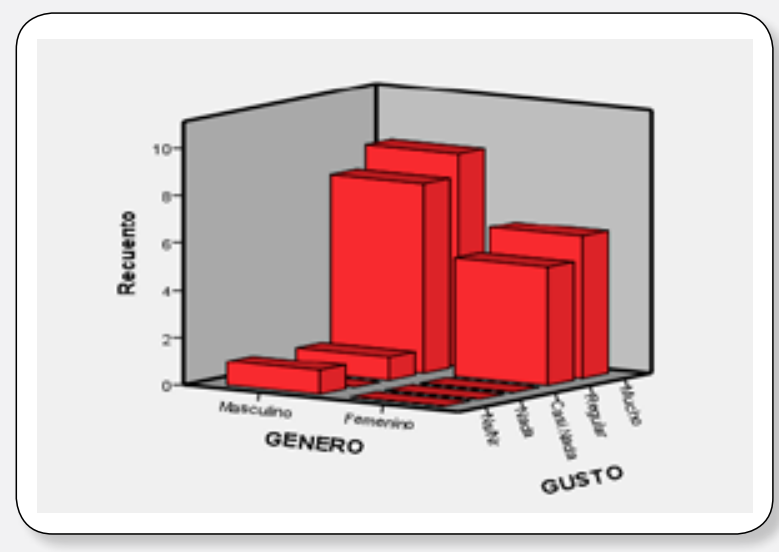

Grafica 1. Género-Gusto

En la tabla 1, se analiza que el $40 \%$ prefiere estudiar el temario; conjunto de los Números, el 30\% la geometría, el 16,7\% las operaciones matemáticas, el $10 \%$ solución de problemas de aplicación, el 3,3\% lógica y razonamiento.

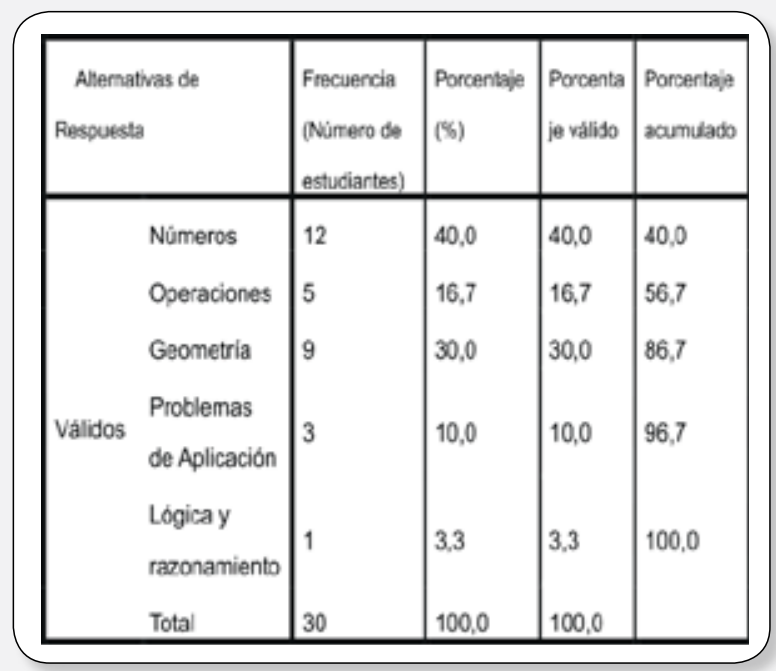

Tabla1. Temario

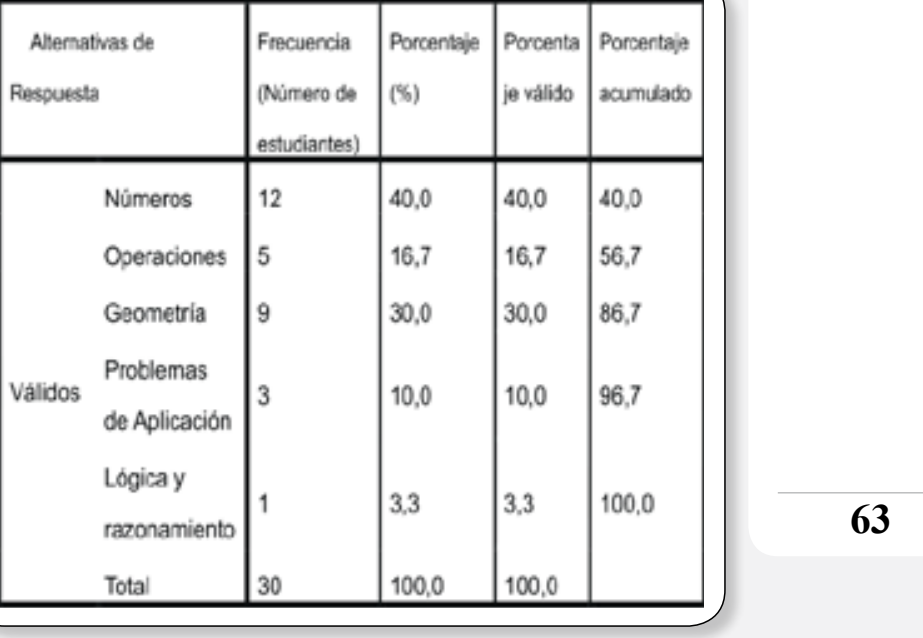
1794-8231

P: 60-65 
No. 1

Enero-Diciembre 2014 ISSN 1794-8231

PP: 60-65

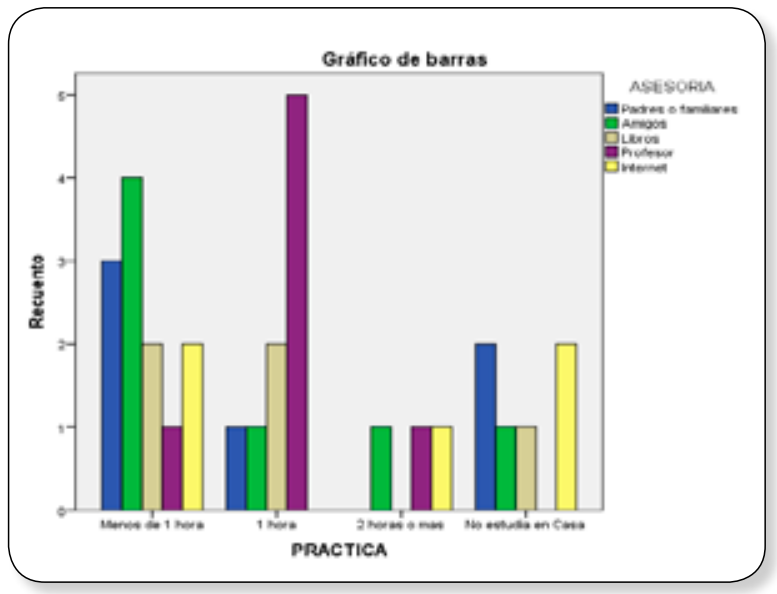

Grafica 2. Práctica de ejercicios

Por más que el estudiante busque asesoría para superar las dificultades de algún tema visto en clase, aún persisten falencias, sin embargo se puede señalar que el $23,3 \%$ busca asesoría en el profesor y el mismo porcentaje busca ayuda entre sus amigos, un $20 \%$ con sus padres o familiares, el $16,7 \%$ consulta sus dudas en la internet (web) y el $16 \%$ restante investiga en libros.

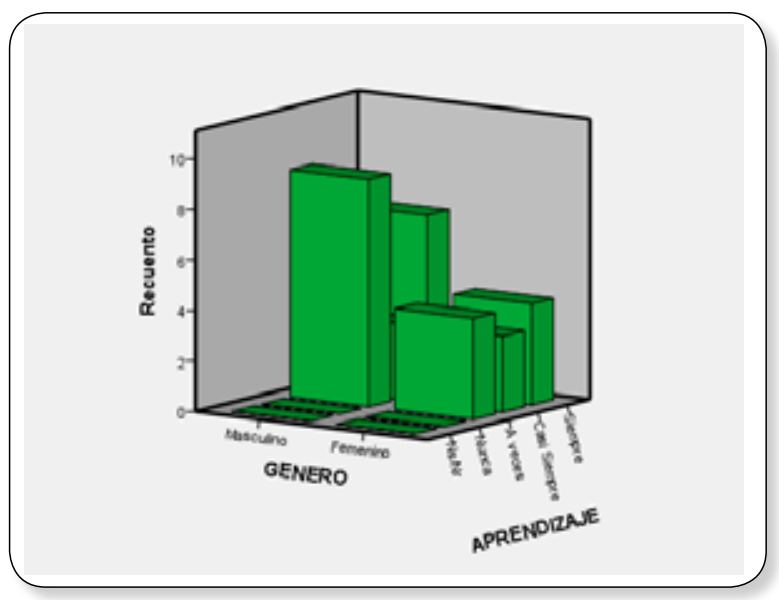

Grafica 3. Aprendizaje

Al analizar la variable aprendizaje, se puede apreciar según la gráfica 3 , que el $43,3 \%$ a veces aprende los temas vistos, el 36,7\% siempre aprende, el 20\% casi siempre atiende, entiende y aprende todo lo relacionado con la temática presentada durante la clase.

Es de destacar, que los estudiantes manifiestan una actitud de aceptación por el área de matemáticas; sin embargo, es función del docente incentivar a los educandos para desper- tar en ellos el amor por el área mediante la aplicación de la teoría transposición pedagógica en el proceso de enseñanza de modo que aumente el porcentaje de los estudiantes que mecanizan, practican y aprenden matemáticas, para que apliquen los conocimientos y destrezas adquiridas en pro del desarrollo de todos los niveles del pensamiento, especialmente el lateral y puedan desenvolverse en diferentes contextos de su vida cotidiana.

\section{CONCLUSIONES}

La matemática hoy, representa un área de interés por los estudiantes en zona de frontera, no obstante deben implementarse prácticas pedagógicas que fomenten más el gusto por estudiarla y entenderla. De igual manera debe incrementarse el número de horas dedicadas a su enseñanza e interacción con otras áreas para aplicarla y motivar el estudio de temas más abstractos de la misma.

La dinámica de frontera acelera la práctica del saber matemático, desde la perspectiva de la operacionalización básica del cambio de divisas, la comercialización de productos con dos tipos de monedas, por los tipos de comercio organizado y el informal (contrabando) dada la rapidez con el actuar y la vulnerabilidad poblacional para superar necesidades básicas de satisfacción NBS según indicadores económicos en Colombia.

El mayor realce radica en tener de primera fuente argumentos valederos que posibiliten detallar parte de la interacción pedagógica específica en matemáticas sobre hábitos de estudio, tiempo, espacio, asesoría, entre otros. Abordar la matemática en un ambiente fronterizo prospectivamente genera cambios en la política educativa interna; a través de la construcción y reingeniería del proyecto educativo institucional con relación directa a métodos, estrategias y evaluación escolar de estudiantes; "como proceso valorativo que pretende la emisión de un juicio para expresar la realidad que subyace en una compleja red de interacciones entre los elementos presentes donde se consideran ideas, intereses, actitudes y emociones"(Revista Quo 2012). 


\section{REFERENCIAS}

Enero-Diciembre 2014

Cardellí, Jorge. Reflexiones críticas sobre

el concepto de Transposición Didáctica de Chevallard Yves. La transposición didáctica. Del saber sabio al saber enseñado. Aique, Buenos Aires, 1991.

Colmenares, Darcy. Revista Educere. SABER-ULA. Mérida. 2004.

Goleman, Daniel. Inteligencia Emocional. Editorial Kairós. Barcelona.1996.

Goleman, Daniel. Inteligencia Social. Editorial Planeta. México. 2006.

Hernández, S, Fernández, C. y Baptista, P. (1998). Metodología de la investigación (2 ed.) México: McGraw-Hill.

Ministerio de Educación Nacional (2003) MEN. Estándares de matemáticas. Bogotá DC.

Serrentino, M. y Pachano Lizabeth. Revista Educere v.9 n.29.SABER-ULA. Mérida. 2005.

www.spssfree.com/spss/multiples $9 . h t m l$ [Consulta, 2012, Noviembre 14]

www.uned.es/revistaestilosdeaprendizaje/ numero_4/Artigos/lsr_4_artículo_4.pdf [Consulta 2013, Octubre 22 ]

www.aportes.educ.ar/sitios/aportes/recurso/ index?rec_id $=107765 \&$ nucleo $=$ matematica nucleo_ense\%C3\%Blanza[Consulta, 2012, Abril 14]

www.virtual.urbe.edu/tesispub/0059404/ cap03.pdf(cita de Hernández et al. (1991)) [Consulta 2012, Mayo 23], Experimental Libertador. Caracas (Venezuela).2012

Artículo El desarrollo del Liderazgo. Revista STATUS QUO de la Secretaría de la Universidad Pedagógica. 